\title{
Model-Based Tracking on Conveyor Belts: Evaluation and Practical Results in the Automotive Industry
}

\author{
Fabian Scheer \\ Daimler Protics $\mathrm{GmbH}$ \\ Fabian.scheer@daimler.com
}

\author{
Moritz Loos \\ Daimler Protics $\mathrm{GmbH}$ \\ Moritz.loos@daimler.com
}

\author{
Markus Neumann \\ Daimler Protics $\mathrm{GmbH}$ \\ Markus.n.neumann@daimler.com
}

\begin{abstract}
Model based tracking (MBT) of painted cars in the automotive mass production on conveyor belts with robots is a challenging task. Many disturbing sources that have an impact on the MBT exist, like the influence of the localized work illumination, the synchronization of the MBT to the conveyor belt, reflections in the paint, variants of the cars and the complexity of the used CAD models. By having such complex systems the mere assessment of the accuracy and stability of MBT approaches by literature can be hard. A real world application is necessary for a better understanding. Therefore, we present the evaluation of MBT for a robotic gap measurement system on painted cars. The influence of local lighting and car paint is analysed in detail regarding the MBT accuracy. To reduce complexity considering the car variants on a production line, we evaluated the MBT with different model setups and show the influence on the MBT results. Regarding MBT in a complex calibrated system that runs twenty-four-seven, a broken or slightly displaced camera should not have a huge impact like a loss of production. For this reason, we present a method to exchange a camera within minutes and without a loss of the overall accuracy. The method relies on a separate test specimen and is evaluated in detail. The presented evaluations can help researchers and the industry to better understand and assess the influence and correlations of different error sources or disturbing factors for the usage of MBT in complex conveyor belt based robotic applications.
\end{abstract}

\section{Keywords}

Application, evaluation, model-based tracking, coated cars, synchronization to conveyor, robotic production line

\section{INTRODUCTION}

By using MBT in complex systems a lot of correlations exist between different influencing factors, like the reflectivity of considered objects, the lighting situation, the optimal selection of the used CAD model or the synchronization to other system components or machines. During research in the literature we found that such relationships are often sparsely considered for MBT approaches and that there is a lack of detailed evaluations of MBT in action. Therefore, we present evaluations of different factors that have an influence to the results of MBT in a complex system. The industrial use case presented in this paper is the automatic measurement of gap points on painted cars on a conveyor belt with a light weight robot. To avoid a crash of the robot with the car, an MBT approach is used that measures the car. On this basis, the robot can correct its teached coordinates with the actual tracking results. Such automated vision based systems play a more and more important role in the context of future manufacturing systems. Various international initiatives like the industrial internet

\footnotetext{
Permission to make digital or hard copies of all or part of this work for personal or classroom use is granted without fee provided that copies are not made or distributed for profit or commercial advantage and that copies bear this notice and the full citation on the first page. To copy otherwise, or republish, to post on servers or to redistribute to lists, requires prior specific permission and/or a fee.
}

consortium, industry 4.0 or smart factories support these ambitions. Against this background, efficient and pragmatic solutions, e.g. maintenance models, for the every day use of such systems are necessary. Based on this motivation, we present a fast method to handle the exchange of broken or displaced cameras without the need of initial measurements for the total system. This method is evaluated in detail and the results are presented and discussed. The presented methods and evaluations in this paper can help researchers and the industry to better assess the behaviour and accuracy of MBT in complex systems and to better understand the impact of different influencing factors and their correlations.

\section{RELATED WORK}

[VP05] gives an overview of monocular model-based tracking approaches in a survey. The authors give a categorized overview to support the selection process which approach may be suitable for a use case, but it is a very rough overview without many details. Indepth evaluations that consider different influencing factors are missing.

[Gar18] summarises state of the art frameworks and datasets for the evaluation of six degrees of freedom object trackers. They limit their method to the criteria of stability, robustness to occlusion and accuracy during challenging interactions. 

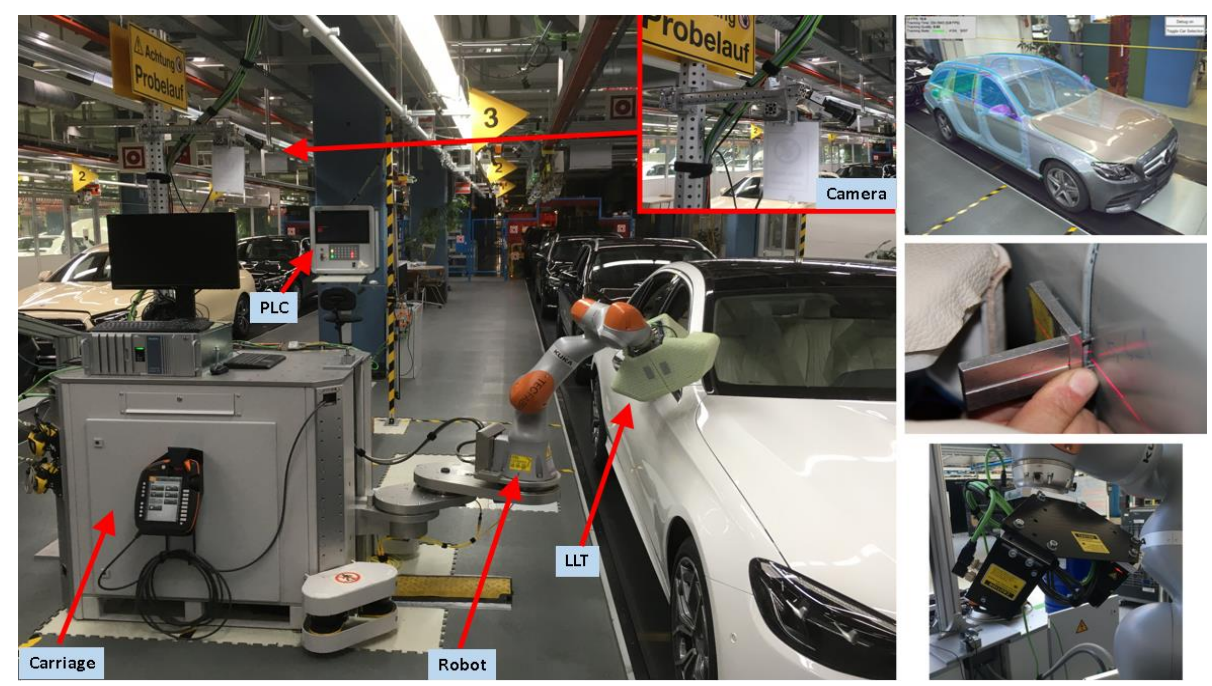

Figure 1: System Components. The camera mount is zoomed. Top right: Overlay of CAD data to video image. Middle right: template for robot teaching. Bottom right: Laser line tool without protective cover.

Several evaluation techniques use fiducials like [Hin11] or a checker board [Wu17]. Therefore, the quality of ground truth information is limited.

In [Hod17] a summary of the state of the art can be found for the evaluation of $6 \mathrm{D}$ pose estimation for textureless objects. They also use fiducials to create ground truth data.

In our former work [Sch20], MBT was evaluated with devices of the measurement technology and an detailed overview of the influence and correlations of several factors was given. A robotic conveyor belt based gap measurement application was shown, but limited to body shells. In this paper our work is extended by considering painted cars that are completely assembled. Section 3.2 recapitulates the used application. Different gloss paints with light reflections are challenging for the MBT. Therefore, different lighting situations in correlation with the car's gloss paint, the optimal CAD model selection and the accuracy of the total system will be evaluated. Detailed results for the gloss paints will be given. Furthermore, a fast method to exchange a camera in the system will be shown and evaluated in detail. It merely introduces a small, acceptable error regarding the system's total accuracy.

In this paper, the MBT approach of [Wue07] that is based on [Com06] and [Vac04] was used for all evaluations. The authors evolved the approach and reached a high degree of maturity. The results in [Sch20] prove that with this MBT a high degree of accuracy and robustness in industrial applications can be achieved. Since we also focus on an industrial use case, this was important in the selection process for the MBT. Only one MBT approach is considered in this paper, due to the great expense for all presented evaluations. To solve the initialization problem, [Wue07] relies on a real camera perspective that closely matches a specified CAD perspective. If both perspectives match, a continuous tracking takes place for subsequent frames and the CAD model can be augmented on the video image (see Figure 1,top right).

\section{System and workflow overview}

Our system uses MBT to track painted cars on a conveyor belt. Tracking results are sent to a light weight robot to navigate to predefined points on the outer shell of a car where gaps are measured. By using the MBT results the robot avoids collisions with a car.

\subsection{Motivation}

Industrial robot applications rely on an initial setup and configuration stage. In this stage, all components are in a defined state. This is necessary to calibrate the different components and put the system into operation. For our use case, a painted car is put on the conveyor belt in an initial position. In this position measurement points on the outer shell of the car are taught to the robot. During production, the painted cars are driven onto the conveyor belt by workers. Therefore, the pose of the cars deviates from the pose of the car in the initial position. Thus, without any pose correction the robot could collide with the cars on the conveyor belt during production. Furthermore, the tire pressure of the cars can lead to the effect that the robots are not measuring the exact gap position on every car. To avoid the described problems a pose correction for every car on the conveyor belt is needed.

\subsection{System setup and workflow}

In Figure 1 the setup of the industrial application is shown. To run the MBT, an industrial PC is used that is integrated into a carriage. On top of the carriage a robust and adjustable camera mount is installed that holds a video camera. A uEye UI-3000SE-C-HQ camera with global shutter, $12 \mathrm{~mm}$ lense and a resolution of 4110x3006 was used for all evaluations in this paper. The light weight robot is also attached to the carriage. To get information about the conveyor 


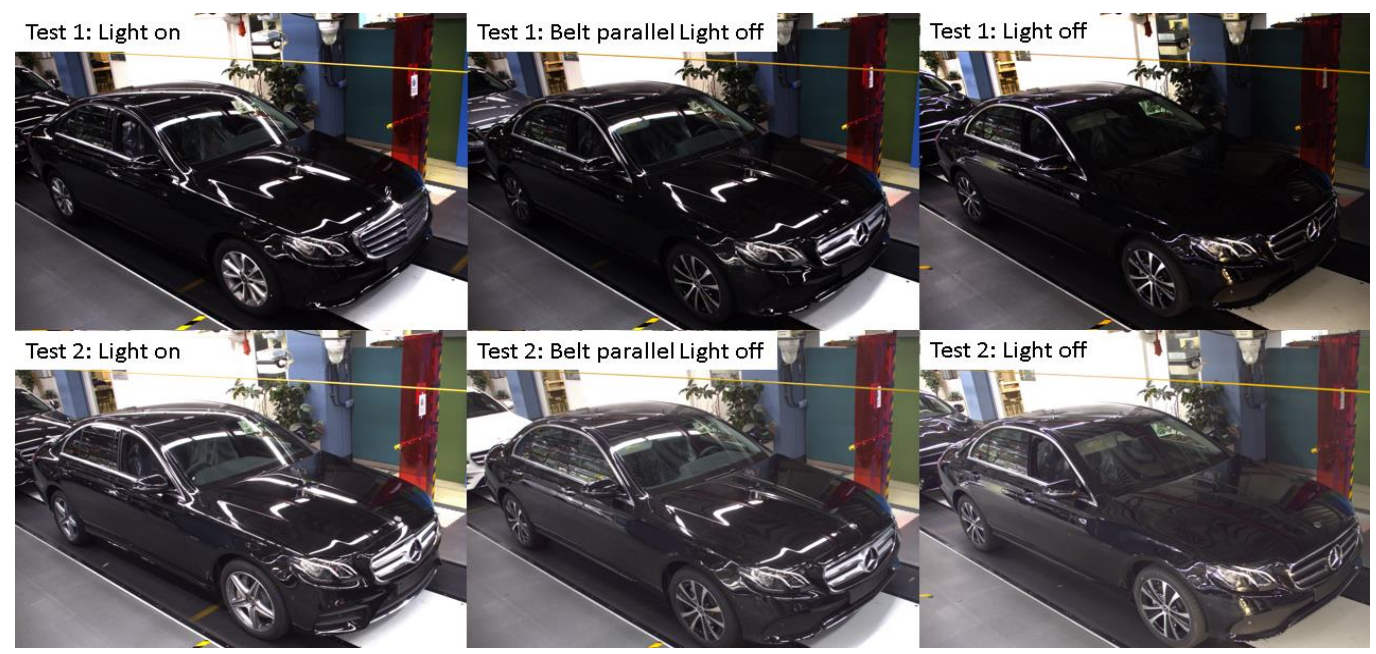

Figure 2: Tests with light turned on, belt parallel lights turned off and light in the station turned off. Top row: Unmodified images. Bottom row: Images with automatic color and contrast correction.

belt movements a rotary encoder is attached under the conveyor belt. A light barrier detects if a car enters the station. All components are connected to a programmable logic controller (PLC) that controls the production station. The PLC on the other hand is connected to the production system and provides information about type, color and variant of the actual car to the system. With this information the correct CAD model can be loaded for the MBT.

To setup the MBT and to teach the robot, the same car is used. The car is brought into a reference position that is fully visible in the view of the camera. This position is called the virtual trigger (VT). Then the painted car is measured with the MBT and the pose is stored as $P_{r e f}$. The increment value of the rotary encoder $I n c_{V T}$ is also stored. During production the passing cars are measured by the MBT at the VT position. From the VT an earlier position in the opposite conveyor direction is calculated and called "first fit". The first fit position lies directly behind the light barrier and is used to start the matching of the edges of the CAD model with the edges detected in the video frames to obtain a continuous tracking for the following frames. When cars during the production pass the VT, the pose $P_{V T}$ is measured by the MBT and the transformation between $P_{r e f}$ and $P_{V T}$ is calculated with $P_{\text {diff }}=P_{r e f}^{-1} * P_{V T}$ and send to the PLC. $P_{\text {diff }}$ determines the shift and twist of the actual car in comparison to the reference car. This information is used further to correct the teached coordinates of the robots measurement points for every car. In this way collisions between the cars and the robot are avoided.

\subsubsection{Synchronization of robot, conveyor belt and model-based tracking}

By using a rotary encoder attached under the conveyor belt the covered distance can be calculated by a conversion factor. The rotary encoder delivers increment values of the belt that can be read by the PLC. If a car enters the station, the light barrier detects it and sets an increment counter to zero. From then on, the PLC sends the actual increment value $I n c_{a c t}$ every 4 milliseconds (ms) to the MBT system. When Inc $c_{a c t}$ is greater than $I n c_{V T}$, the car has reached the VT and a timestamp $t_{a c t}$ is stored in the MBT system. The MBT system sets a timestamp for every calculated pose. Thus, the last pose $P_{t 1}$ before timestamp $t_{a c t}$ and the first pose $P_{t 2}$ after $t_{a c t}$ can be used together with the time difference $t_{\text {diff }}$ to calculate the interpolated pose $P_{V T}$ at the virtual trigger position. With $P_{V T} / t_{\text {diff }}=$ factor , the slerp function can be used to interpolate between the poses. In this way $P_{V T}$ at time $t_{a c t}$ is calculated and can be compared directly with the reference pose $P_{\text {ref }}$. Because the increment values are merely sent every $4 \mathrm{~ms}$, an additional error of approximately $0.3 \mathrm{~mm}$ can occur with a conveyor speed of $75 \mathrm{~mm}$ per seconds that was used in the experiments.

In the configuration stage of the production station the transformation of the car to the robot base and the conveyor belt direction is measured with a high precision measurement device like a Faro laser tracker [Far21] or a Creaform metra scan [Cre21]. By using these information together with the pose correction $P_{\text {diff }}$ and the conveyor increments including the conversion factor, the robot can predict the exact car position and respective measurement points. Thus, the robot can approach each calibrated measurement point on the car correctly without any collisions.

\subsection{Error sources}

Several error sources have an influence on the accuracy of the total system in this complex application. A detailed evaluation regarding the single error sources can be found in [Sch20]. We sum up the most important ones under an assumption of $2 \sigma$ :

- MBT errors: $0.7 \mathrm{~mm} \pm 1.5 \mathrm{~mm}$

- MBT-conveyor synchronization: $1.7 \pm 1.5 \mathrm{~mm}$

- Robot to conveyor synchronization: $\pm 0.5 \mathrm{~mm}$ 

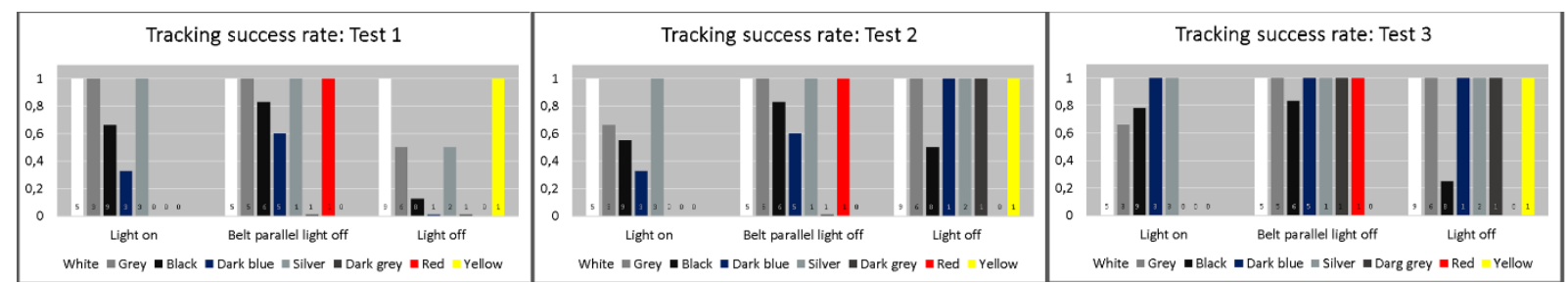

Figure 3: Results of the light tests for different gloss paints.

- Robot calibration of gap points: $\pm 0.5 \mathrm{~mm}$

- Production tolerance of test object $\pm 0.5 \mathrm{~mm}$

- Calibration conveyor direction

- Calibration robot to test object

In addition, several other factors have an influence on the MBT results, like the localized work illumination, the different colors of the car paint or the selection of the CAD parts that are used for MBT.In the following sections these factors are evaluated in detail. An accuracy analysis for such a complex system is difficult, since many factors have an influence on the result. In [Sch20] separate factors are evaluated. But at least the accuracy of the final system output is essential. Therefore, we also present an evaluation of the total accuracy of the system. Thereby, the laser line tool at the robot head is used to determine how precise the robot can approach the several measurement points on the outer shell of painted cars.

\subsection{Influence of illumination}

In [Sch20] the influence of lighting and temperature over time on MBT for body shells was evaluated. For the use case of painted cars the influence of lighting is bigger due to reflections or the influence of car paint to the edge detection and matching of the MBT. As first evaluation criterion, we used the tracking quality. This is an output of the used MBT that describes how many edges of the CAD model could be matched to the edges in the respective camera image. A factor of 0.8 for examples denotes that 80 percent of the CAD model edges could be matched.

The localized work illumination for production facilities is built up considering a norm. Mostly fluorescent tubes are used. Fluorescent tubes that are aligned parallel to the conveyor belt can have a negative influence to the MBT. The reflections of the tubes can cause the detection of an edge by the MBT that is similar to edges coming from gaps between car parts. If these edges lie in the surrounding area of a correct edge a mismatch can occur. The effect is visible in Figure 2. A light reflection lies in the direct surrounding of the gap between the car hood and the car wing. Another observed issue was that white areas, e.g. walls, in the video image can outshine image regions. This can be seen in Figure 2. The background wall outshines the contour edges of the car roof. This can lead to a bad initial match of the MBT edges. Another problem concerning a correct MBT matching process is the bad contrast between the black colored belt and the underbody for cars with dark or black gloss paint. To overcome the described problems we tested different settings in our evaluation. First, we tested three light situations: All lights turned on, belt parallel lights turned off and all lights turned off. We call this test "test 1"(see Figure 2). Then we modified the camera images with an automatic color and contrast correction. This is "test 2". In "test 3" we modified the tracking parameters. Thresholds were modified to detect more edges in the video image and the initial tracking value was lowered. The initial tracking value relies on the tracking quality value that describes in percentage from when on a matching can take place, e.g. try a match if 50 percent of the edges match. The results are listed in Figure 3.

For this test we defined a tracking success rate. Only results with a tracking quality greater than 0.55 and a jitter smaller than $5 \mathrm{~mm}$ were considered as a successful tracking. The decision was based on the observation that with results smaller than 0.55 the MBT more often runs into local minima. The success rate is marked on the left scale and describes how many of the evaluated cars could be successfully

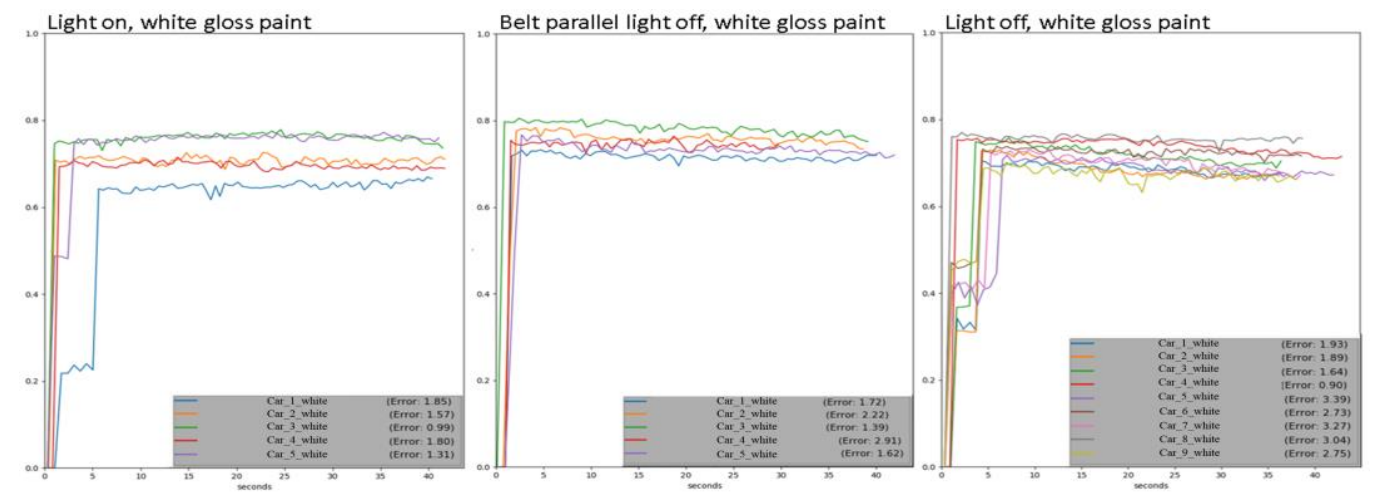

Figure 4: Tracking quality results for cars with white gloss paint over time in different lighting situations. 


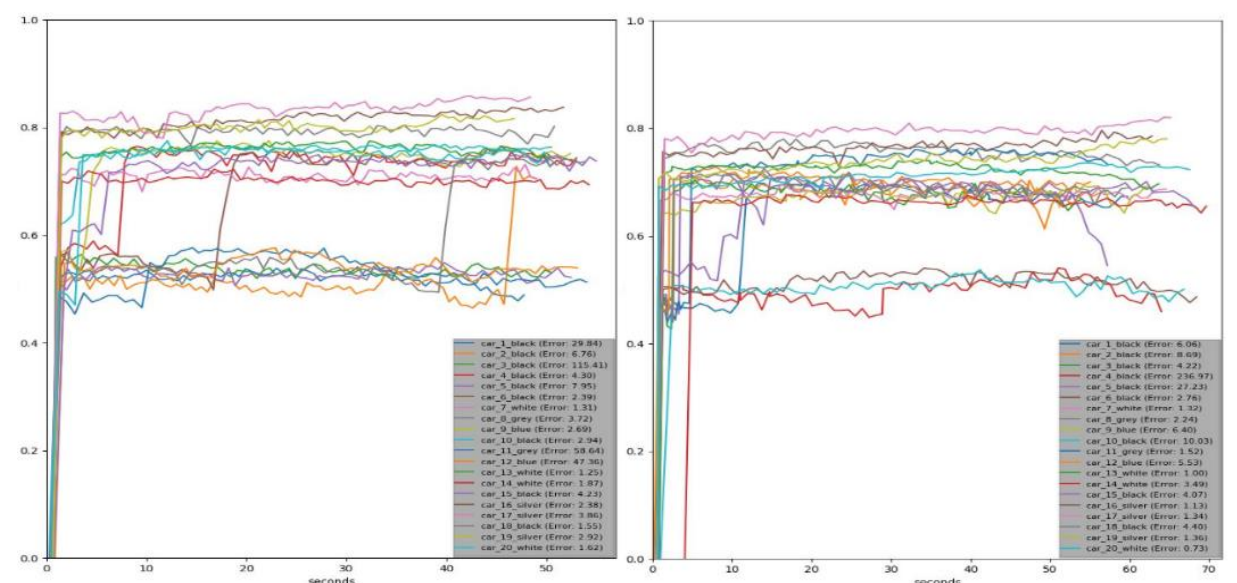

Figure 5: Results over time for different models: Left: Common parts. Right: Full model with all variants.

tracked after the criteria described above. The gloss paint of the cars is depicted in the bottom line and corresponds to the bars. The numbers on the bottom of the bars show how many cars per color were considered. This means: If 5 cars were considered per gloss paint and all are tracked successfully the bar goes up to a value of one. If only three out of five are tracked successfully the bar goes up to 0.6.

The most significant findings are that the automatic color and contrast correction improves the success rate for the situation where all lights are turned off. Otherwise, no big differences can be found between "test 1" and "test 2". The best overall result can be found when the belt parallel lights are turned off in "test 3". Further tests considered the behavior of the MBT over time. In Figure 4 the results of the tracking quality for a moving white car can be seen for a period of 35 seconds. The best results were also achieved for the lighting situation with belt parallel lights turned off. This lighting achieves the best matching rates between $\mathrm{CAD}$ and video image edges from the beginning of the period. Under the other light situations the MBT needed some time until the matching yields the best rates and the tracking quality is generally lower. Due to our findings we kept the light and tracking parameter settings that achieved the best results for our further evaluations.
In Figure 4 we also listed an average error. We estimated for all poses of a car during the period a best fitting line with RANSAC and computed the average deviation along this line. This can serve as a measure to check how stable the MBT calculates the car poses, because due to the conveyor belt setup it can be expected that the cars poses lie on a straight line according to the belt direction.

Throughout the evaluation we found that completely black cars are a challenge for the MBT. Especially the low contrast to the conveyor belt can have a disturbing influence on the MBT. Due to this, we conducted another evaluation with additional lights. Four spotlights were used to illuminate the gap between the belt and the underbody and another spotlight behind the car that illuminates the background to improve the contrast (see Figure 7). Furthermore, the gamma correction of the video camera was turned on to further improve the contrast. For black cars we found that the additional lighting improves the tracking quality value, meaning more edges could be matched. Approximately 10 percent more edges could be found (see Figure 7, top row). By turning on the gamma correction with no additional lighting we achieved nearly the same tracking quality values (see Figure 7, middle row). Turning the light and the gamma correction on, a tracking quality value of 0.79 was achieved (see Figure 7, middle row). Since additional
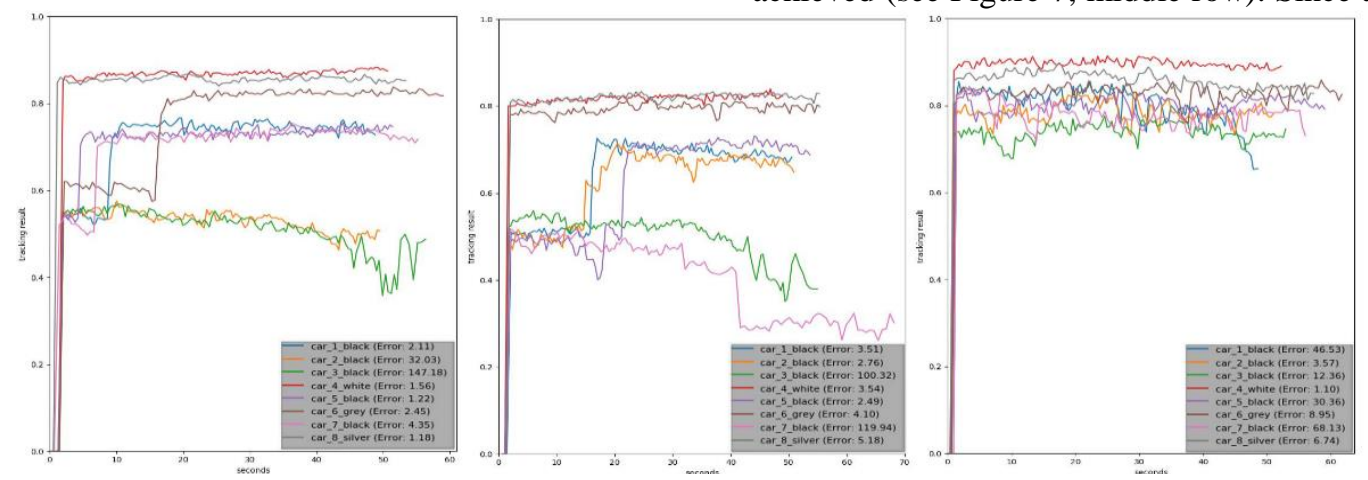

Figure 6: Tracking quality results over time for CAD models of cars with common parts over all variants (left), of a half car model (mid) and of the outer contour of common parts(right). 
lights tend to outshine image regions a test for white gloss paint was needed to exclude any unwanted side effects. For such a car we achieved opposite results. With gamma correction a result of 0.84 was obtained. With lighting turned on the tracking quality dropped to 0.78 (see Figure 7, bottom row).

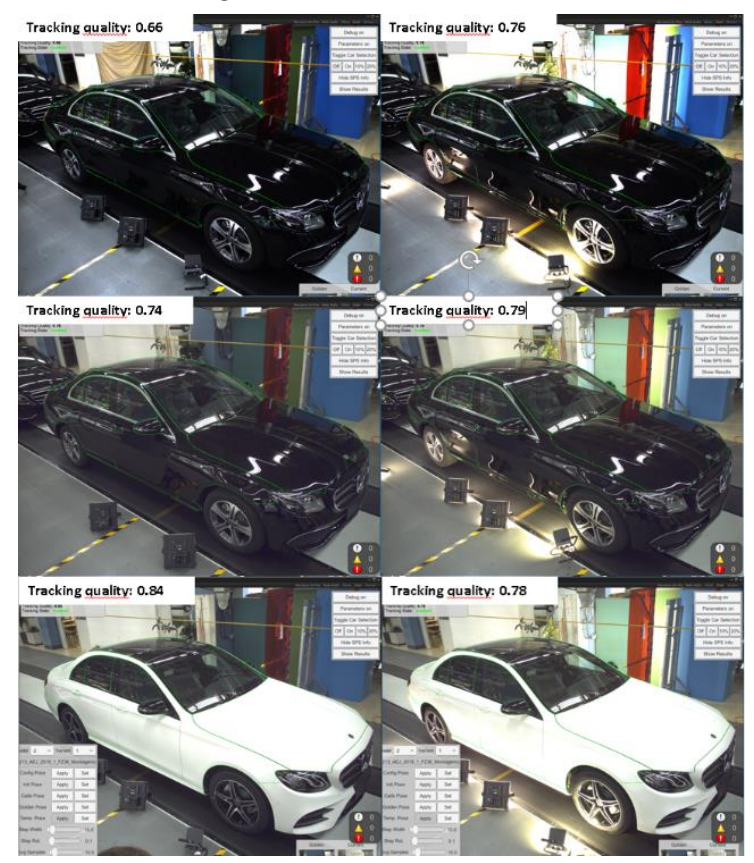

Figure 7: Images of the test with additional lighting and gamma correction turned on.

With outshined surfaces MBT has problems in finding edges correctly. This test was also conducted for other gloss paints. In general, we found that the gamma correction improves contrasts and reduces outshined surfaces in the images in a way that the MBT can achieve a better matching. We also found that the gamma correction has a similar effect on the tracking quality than the color and contrast correction. Therefore, we just use the gamma correction and avoided the additional color and contrast correction. Since we don't want to use special adjustments of the MBT parameters, the camera settings and the lighting situation for every possible gloss paint, the best solution to work with is one setting that fits it all. According to the results above the best solution regarding a cost-benefit analysis can be achieved with the belt parallel lights turned off, the gamma correction of the camera turned on and the usage of the optimized parameters for the MBT.

\subsection{Evaluation of different CAD models}

Considering all car variants produced on a production line, there is a high complexity of the used CAD models. Reducing this complexity would lead to a more manageable situation for the usage of MBT in industrial settings. Therefore, another evaluation was conducted that compares the usage of CAD models with all variant parts, of common parts over all variants only and of the outer contour of common parts only. Common parts are for example the chassis, the hood, the doors, the trunk, the roof, the windows, the front shield, the car wings, etc..

In the production every station meter costs. Reducing the station length by shortening the view space of the camera would reduce such costs. On that account, we evaluated the usage of a half car model only. This evaluation was conducted at the beginning of all of our tests. Therefore, the settings described in the previous section 3.4 were not used. This can be seen in the generally lower tracking quality values and higher jitter around the fitted RANSAC vector for all tests in this evaluation (see Figure 6).

In Figure 6 the tracking quality over time is shown for common parts that can be used for all car variants (left), a half car model (middle, the front half of the car was used) and for the outer contours (only contour edges used for the MBT) of common parts of all car variants. Considering the tracking quality value, the number of matched edges for the outer contour achieves the best results, followed by the common parts. The results for the half car model are the worst. But setting these values in correlation to the fitted average vector with RANSAC, the situation looks different. The poses of the common parts test slightly vary around the fitted average vector. For the half car model the deviations to the RANSAC vector increase and for the outer contours we found a big jitter and even big jumps in the single poses in relation to the straight fitted vector of poses with RANSAC. The tests also contained outliers as can be seen on the deviations in form of errors to the RANSAC vector. The tests were conducted before the illumination tests in section 3.4. By using the results of section 3.4, we observed that such outliers are drastically reduced. Finally, the common parts achieved the best results in terms of stability and robustness, even though a smaller amount of edges can be matched.

In another test, common parts are compared to the CAD model that contains the full car variants CAD data, for example with special parts for a car line like the sill, driving mirror, radiator grill etc.. In Figure 5 the results are shown. The results are ambivalent. For some cars the tracking quality with the common parts are better and for other cars the full CAD model gets better results. A handful of cars benefit from the full CAD model, but don't represent the majority of cars. With the common parts slightly more outliers are present considering RANSAC. Since the results aren't that clear and overall both method generate stable and robust average results, we decided to continue with the method of using common parts. This is because the model preparation, including part search in the database, data export and converting the data into the necessary formats takes time. Under a cost-benefit analysis, using the common parts makes sense in 


\begin{tabular}{|c|c|c|c|c|c|c|c|c|c|c|c|}
\hline & Quantity & P1 X & P1Y & P2 $\mathrm{X}$ & $\mathrm{P} 2 \mathrm{Y}$ & P3 $x$ & P3 Y & P4 Y & P4 Z & P5 Y & P5 Z \\
\hline Black & 27 & $0,99 \pm 4,21$ & $-1,1 \pm 3,79$ & $1,76 \pm 4,32$ & $-3,13 \pm 3,52$ & $-1,29 \pm 6,78$ & $-1,78 \pm 4,05$ & $-1,88 \pm 3,79$ & $0,52 \pm 6,41$ & $0,33 \pm 4,37$ & $1,44 \pm 3,68$ \\
\hline White & 68 & $-0,82 \pm 5,06$ & $0,7 \pm 2,91$ & $-1,2 \pm 3,90$ & $-1,54 \pm 2,81$ & $-3,24 \pm 5,21$ & $-0,41 \pm 2,91$ & $0,71 \pm 2,97$ & $-1,02 \pm 6,12$ & $0,74 \pm 2,62$ & $0,41 \pm 3,04$ \\
\hline Obsidian black & 55 & $0,12 \pm 4,14$ & $-0,77 \pm 4,16$ & $0,91 \pm 4,42$ & $-2,91 \pm 3,28$ & $-1,99 \pm 4,89$ & $-1,42 \pm 3,54$ & $-1,7 \pm 4,24$ & $1,4 \pm 6,14$ & $0,59 \pm 4,00$ & $1,77 \pm 3,10$ \\
\hline Diamant white & 5 & $-1,12 \pm 4,43$ & $0,25 \pm 1,01$ & $-1,69 \pm 4,41$ & $-1,92 \pm 1,76$ & $-3,33 \pm 5,13$ & $-1,19 \pm 2,40$ & $0,51 \pm 2,34$ & $0,21 \pm 5,39$ & $0,12 \pm 2,25$ & $0,66 \pm 2,92$ \\
\hline Graphite grey & 12 & $-0,91 \pm 5,57$ & $-0,03 \pm 2,66$ & $-0,36 \pm 4,43$ & $-2,53 \pm 2,35$ & $-2,86 \pm 7,08$ & $-1 \pm 2,49$ & $-1,31 \pm 3,44$ & $1,65 \pm 6,89$ & $0,78 \pm 2,39$ & $2,27 \pm 3,82$ \\
\hline Covansit blue & 13 & $-0,1 \pm 5,10$ & $-0,98 \pm 5,41$ & $0,3 \pm 4,89$ & $-2,29 \pm 4,58$ & $-1,68 \pm 7,86$ & $-0,6 \pm 5,07$ & $-2,14 \pm 6,52$ & $2,39 \pm 6,36$ & $1,41 \pm 5,25$ & $1,06 \pm 3,44$ \\
\hline Hightech silver & 37 & $-2,62 \pm 4,31$ & $1,08 \pm 2,62$ & $-1,2 \pm 2,40$ & $-1,49 \pm 2,26$ & $-3,64 \pm 4,00$ & $-0,23 \pm 2,43$ & $0,04 \pm 3,32$ & $0,2 \pm 7,03$ & $1,3 \pm 2,62$ & $0,87 \pm 2,79$ \\
\hline Selenit grey & 40 & $-2,82 \pm 5,00$ & $0,63 \pm 3,22$ & $-1,13 \pm 3,62$ & $-1,95 \pm 3,01$ & $-4,55 \pm 5,24$ & $-0,29 \pm 3,50$ & $-0,56 \pm 3,07$ & $0,37 \pm 6,17$ & $1,28 \pm 3,80$ & $1,96 \pm 2,67$ \\
\hline
\end{tabular}

Table 1: Results for the total system accuracy for different gloss paints.

regard to an industrial application. Furthermore, this approach is safeguarded by the evaluation in the next section, where the accuracy of the total system is evaluated. But if an application or approach has problems reaching given accuracy requirements, our findings confirm that it may be better to use the full CAD model for MBT of painted cars.

\subsection{Evaluation of the system accuracy}

The determination of the accuracy of a complex system can be challenging. Correlations exist between single error sources and further influencing factors, like lighting, CAD model selection or the gloss paint. In section 3.3 the single error sources are described that were evaluated in [Sch20]. For painted cars further factors have to be considered, whose influence was evaluated in the previous sections. With these evaluations researchers and the industry can better understand the relationship and possible effects of single components, influencing factors and their complex interaction. But in the end the total error of a complex system is essential to assess the accuracy, robustness or applicability.

Therefore we use the same method to determine the total accuracy of the system as described in [Sch20]. The laser line tool (LLT) that is mounted on the robot head to measure gaps is also used to measure the accuracy of how exactly the robot approaches a measurement point. The LLT consists of two laser line scanners. They are arranged in a special mount and specifically aligned to create one large laser scan line. In Figure 1 the LLT is shown. If the robot measures a gap point at a car the deviation of the gap midpoint to the LLT midpoint can be measured (the output is a coordinate on the laser line and the distance to the surface). This error is given in 2D LLT coordinates and serves as a metric to assess the total accuracy of the system. With this evaluation criterion it can at least be determined how precise the robot can reach a single measurement point and gives us an assessment of the operational fitness of the MBT for this kind of application scenario.

The measurement points are taught to the robot in the initial position. The LLT is aligned perpendicular to the normal of each gap and precisely aligned with a special template (see Figure 1). Little bridges on the backside guarantee a tight fit into a gap. Then the large laser line is orthogonally aligned to the template by an expert and the robot's position is stored. To overcome possible errors in this manual process, a software offset based on the measurements is calculated. It corrects systematic deviations and ensures a tight orthogonal alignment of the LLT's laser line to a gap. With the car in the reference position for the MBT, the direction of the conveyor belt and the transformation between the robot base and the painted car is measured with a high precision measurement device, like a Faro laser tracker. All these values together with the conveyor belt increments for the reference position are used by the robot in the conveyor belt synchronous mode to predict the position of a car for the actual belt increment values. The MBT measures the actual car at the VT (see section 3.2) and calculates a pose correction in comparison to the reference car. With this pose correction and the predicted car position the calibrated measurement points can be corrected in robot coordinates for the considered car.

To evaluate if the accuracy is suitable to avoid collisions of the robot with the cars and to fulfill the accuracy requirements, an evaluation for five measurement points on 261 cars was conducted. Measurement point $\mathrm{P} 1$ is at the middle of the gap between the car front door and the car wing. P2 is on the middle of the gap between the back and the front door. P3 is at the middle of the gap between the back door and the rearward car wing. P4 is at the middle of the gap between the hood and the car wing. And the last measurement point P5 is at the middle of the horizontal bottom gap of the tank cap. The results are shown in Table 1. As explanation: The origin of the car coordinate system is at the center of the front suspension, whereby the $\mathrm{X}$-axis points along the cars main axis to the back wheels, the $\mathrm{Y}$-axis points to the right front wheel and the $\mathrm{Z}$-axis points up. Since the LLT has to be aligned orthogonally to a gap to perform measurements and delivers $2 \mathrm{D}$ results, vertical gaps are measured with $\mathrm{X}$ and $\mathrm{Y}$ coordinates, according to the cars coordinate system, and horizontal gaps are measured with $\mathrm{Y}$ and $\mathrm{Z}$ coordinates. We evaluated the mean value and the standard deviation of the difference between the measured gap and the measured LLT midpoint. In sum 261 cars were considered. In Table 1 only 257 are shown, because the additional cars had four separate colors, whereby no mean value and standard deviation could be calculated. In Table 1 the results are listed under an assumption of a Gauss distribution with $2 \sigma$. Thus, approximately $95 \%$ of all measurements should lie in 


\begin{tabular}{|l|c|c|c|c|c|c|}
\hline & \multicolumn{5}{|c|}{ Cars clustered by brightness } \\
\hline & Bright & White & Bright without white & Dark & Black & Dark without black \\
\hline Car count & 112 & 73 & 39 & 149 & 82 & 67 \\
\hline P1 X & 5,04 & 5,02 & 4,27 & 5,41 & 4,23 & 5,66 \\
\hline P1 Y & 2,80 & 2,82 & 2,70 & 4,11 & 4,05 & 3,87 \\
\hline P2 X & 3,48 & 3,94 & 2,39 & 4,72 & 4,45 & 4,19 \\
\hline P2 Y & 2,60 & 2,75 & 2,28 & 3,45 & 3,37 & 3,30 \\
\hline P3 X & 4,81 & 5,20 & 3,90 & 6,36 & 5,62 & 6,67 \\
\hline P3 Y & 2,75 & 2,90 & 2,42 & 3,86 & 3,73 & 3,70 \\
\hline P4 Y & 3,16 & 2,93 & 3,34 & 4,22 & 4,10 & 4,22 \\
\hline P5 Y & 6,49 & 6,10 & 6,96 & 6,40 & 6,28 & 6,53 \\
\hline P5 Z & 2,65 & 2,61 & 2,55 & 4,09 & 4,12 & 3,92 \\
\hline
\end{tabular}

Table 2: Results for the total system accuracy grouped by brightness.

the range $\mu \pm 2 \sigma$. Generally, the standard deviation in the $\mathrm{X}$ direction are higher, because of the errors from the conveyor belt synchronization with the robot and with the MBT. Another finding is that for the $\mathrm{Z}$ coordinate of $\mathrm{P} 4$ the deviation for all considered gloss paints is constantly higher. This is partially caused by the error in the $\mathrm{z}$ coordinate for the point itself and in addition by the synchronization to the conveyor belt in $\mathrm{x}$ direction. Since P4 is on the curved hood, errors in the synchronization have an influence on the position where the gap is measured. This results in higher deviations of the $\mathrm{Z}$ coordinate. But nevertheless, the mean values are mostly in the range of up to two millimeters. Further findings are, that darker colors have a slightly higher standard deviation. This is plausible, because darker colors are more challenging for the MBT. From Table 1 it may be not that clear, but if the results for different colors are grouped by brightness, the difference gets much clearer. This can be seen in Table 2 . The results represent the values for two times the standard deviation $(2 \sigma)$. 261 car were considered. Referring to the colors in Table 1, black, obsidian black, graphite grey, covansit blue and selenit grey were assigned to the cluster "dark". White, diamant white and hightech silver were assigned to the cluster "bright". In sum 12 different gloss paints were considered. By separating colors like pure white or pure black, that are more challenging to the MBT (white gloss paint due to the outshining), and showing clusters without these cases, other findings are that for the bright colors without white the deviation slightly decreases; but for dark colors without black in the opposite case a slight tendency is recognizable that black may not be the most challenging color. Other dark colors seem to have a slightly more increasing influence on the standard deviation.

Considering the mean values, the total system achieves results, mostly varying in the range of up to two mm. In comparison of all values the point P3 achieves the worst results over all colors for the $\mathrm{X}$ coordinate. Since this point is one of the last measured points, rotational errors in the system could increase the results. The results for the tank cap with P5 however foil this assumption. P5 is measured as the last point, but neither shows very high results for the mean value, nor the standard deviation. Thus, we don't expect the MBT or the measured belt direction and transformation between the car and the robot as the reason for this effect. The most likely cause seems to be that during the manual calibration of the point by the robot, an error was introduced.

Overall the total system achieves results for the five measurement points and for all gloss paints that are suitable for automatic gap measurements. For 80 coordinate values listed in Table 1, 71 were under an total error of $8 \mathrm{~mm}$, under an assumption of a Gaussian distribution with $\mu \pm 2 \sigma$. Six values lied under $9 \mathrm{~mm}$ and three under $10 \mathrm{~mm}$. From these nine values above $8 \mathrm{~mm}$, six are related to the $\mathrm{X}$ coordinate of point P3. The possible reasons for this behavior were described above. Finally, the laser scan line has a total length of several centimeters. Under these circumstances, the evaluation showed that even with the worst case accuracy of up to $10 \mathrm{~mm}$ in some cases, gaps can be measured by the LLT without any difficulty.

\section{Evaluation of a pragmatic camera maintenance concept}

The setup of the system requires the measurement and teaching of a reference car. In case of a camera defect, this procedure has to be repeated in a production free time slot. It is also necessary for a camera position change. Normally, the occurrence of the second case is rather unlikely due to the robust and tight camera mount, but if an object or person crashes with the camera mount, it cannot be completely excluded that the position of the camera slightly changes. To overcome these limitations we present a method to compensate such errors without the need of a new initial measurement. Therefore, we use a special test specimen that is shown in Figure 8. During the car measurement in the reference position the test specimen is setup and also measured with the MBT. If no camera position change occurred, the measurement of the test specimen by the MBT can also be conducted after the initial measurements. With these measurements, the transformation between the reference car and the test specimen can be calculated, as shown in Figure 8 (left). If the camera is exchanged due to a defect or if the camera is displaced, the specimen is set up again and measured with the MBT. By using the transformation $T_{\text {Spec }}^{\text {Cam } 2}$, the former calculated transformation between the car and the specimen $T_{\text {Spec }}^{\text {RefCar }}$ and the pose of the actual car during production $T_{\text {Current }}^{\text {Cam } 2}$, the transformation between the actual car and the car in the reference position out of 

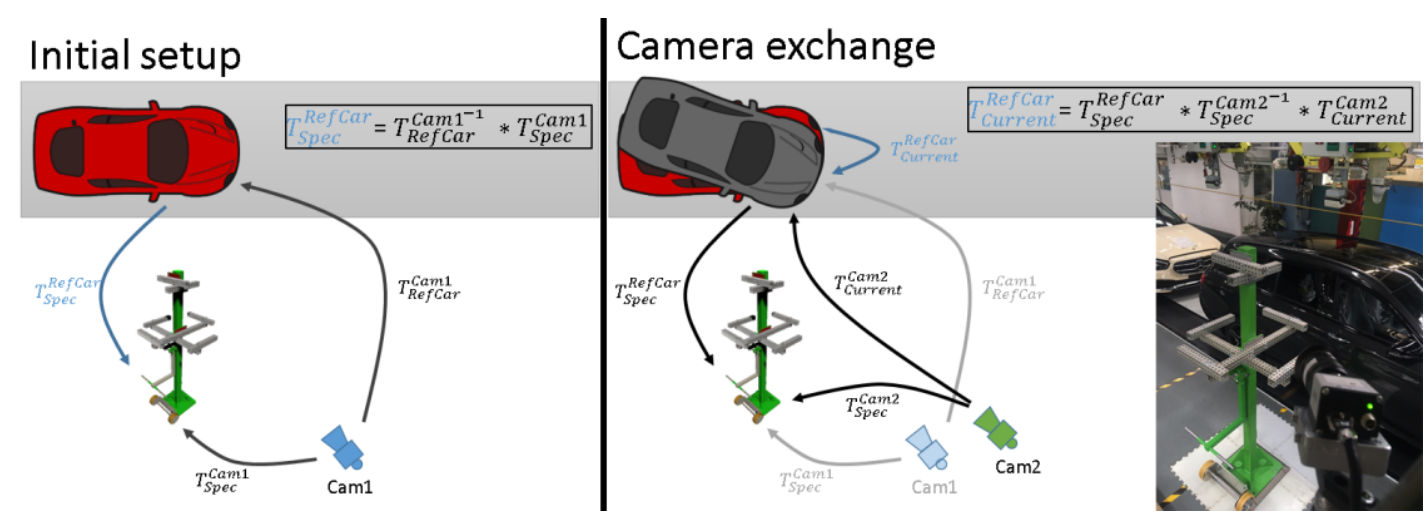

Figure 8: Transformations for the usage of an additional test specimen for the pose correction calculation.

sight of the new or modified camera can be calculated. This transformation can be further used as the pose correction for the robot (see Figure 8, right).

\subsection{Evaluation camera exchange}

The approach to handle a camera exchange or camera displacement in a pragmatic way without the need for time consuming initial measurements was evaluated in several tests. The CAD model of the specimen was created by a 3D scan with a high precision GOM 3D scanner [GOM21].

\subsubsection{Repeatability of the installation}

\begin{tabular}{|c|c|c|c|c|c|c|}
\hline & $\mathbf{X}$ & $\mathbf{Y}$ & $\mathbf{Z}$ & RotX & RotY & RotZ \\
\hline $\boldsymbol{\mu}$ & 1071,58 & $-998,44$ & 2202,91 & 44,03 & 55,82 & 61,35 \\
\hline $\boldsymbol{\sigma}$ & 0,10 & 0,14 & 0,14 & 0,005 & 0,007 & 0,005 \\
\hline Max diff to $\boldsymbol{\mu}$ & 0,21 & 0,29 & 0,26 & 0,011 & 0,017 & 0,010 \\
\hline
\end{tabular}

Table 3: Results for repeated setup of the specimen.

The specimen is mounted on the ground of the factory. Therefore, a pinned fitting was used to overcome problems that may arise by screw tightening. In the test the specimen was setup and dismantled ten times and measured with the MBT. We calculated mean, standard deviation and maximum difference to the mean value. Results are shown in Table 3 and show that with this installation method the standard deviation is very low and the biggest deviations are in the range of 0.2 to $0.3 \mathrm{~mm}$ for the translation and about $0.01^{\circ}$ for the rotation. Thus, the setup and mounting of the test specimen is not a big error source.

\section{1 .2 Verification of new pose correction
\begin{tabular}{|c|c|c|c|c|c|c|}
\hline & $\mathbf{X}$ & $\mathbf{Y}$ & $\mathbf{Z}$ & $\mathbf{R o t} \mathbf{X}$ & $\mathbf{R o t Y}$ & RotZ \\
\hline Mean difference & 0,030 & $-0,138$ & 0,346 & $-0,004$ & $-0,012$ & $-0,006$ \\
\hline
\end{tabular}}

Table 4: Mean of pose correction differences.

To compare the new pose correction calculation with the test specimen with the old pose correction calculation, another test was conducted that investigates the influence of the further measurement of the specimen with the MBT. First the specimen was measured with the MBT. Then, the pose correction for 27 cars on the belt was calculated with both methods under the usage of the same camera. Then the difference between the pose correction transformations was calculated. In Table 4 the mean of these differences is shown. The results only differ in the first decimal place for the translation and in the second decimal place for the rotation values. Thus, in a direct comparison the pose correction with the help of the test specimen introduces a very small error.

\subsubsection{Camera exchange and position change}

In the next step, three tests with different uEye UI3000SE-C-HQ cameras were conducted to test the camera exchange. First, camera 3 (cam3) was used and 30 cars were measured in the same way described in section 3.6. These measurements were used as a reference to compare the further test results. Instead of five measurements points we neglected the tank cap and used four points only. These were the same as in the evaluation in section 3.6. In test 1.1 cam 3 was exchange with cam 1 and then 20 cars were measured. Then cam1 was exchanged with cam 2 and 22 cars were measured. Afterwards cam 2 was exchanged with cam 3 again and 23 cars were measured. In test 2.1 cam1 was used again and we simulated a crash of an object or person with the camera mount by rotating the camera a bit within the ball joint of the mount. 20 cars were measured subsequently. Then, cam1 was exchanged by cam 2 and 20 cars were measured. After this, cam 2 was exchanged by cam 3 and 20 cars were measured. For test 3.1 cam 3 was used and the camera position was modified somewhat stronger. Therefore, the cameras were rotated with the ball joint and also translated by moving the horizontal and vertical elements of the camera mount for some centimeters. With this setting 19 cars were measured. In test 3.2 the procedure was repeated and the camera position was further changed. Then, 12 cars were measured. The test results are shown in Table 5. We calculated the mean value and then the difference to the mean value of the reference test (see above). Therefore, the results in Table 5 are given as $\mu$ diff $\pm 2 \sigma$. The results show the difference of the mean of the new method with test specimen in comparison to the calculation method from section 3.6 and two times the standard deviation. For the mean difference, the majority of the results lie in the range of up to $1 \mathrm{~mm}$. Only a few are in the range of 2 to $4 \mathrm{~mm}$. The results for two times the standard deviation are comparable to the results of section 3.6 with a very slight tendency of increasing values. Since the laser line of the LLT is several centimeters long, 


\begin{tabular}{|c|c|c|c|c|c|c|c|c|}
\hline & Test 1.1 (Cam1) & Test 1.2 (Cam2) & Test 1.3 (Cam3) & Test 2.1 (Cam1) & Test 2.2 (Cam2) & Test 2.3 (Cam3) & Test 3.1 (Cam3) & Test 3.2 (Cam3) \\
\hline Status & \begin{tabular}{|l|} 
Cam change \\
\end{tabular} & Cam change & Cam change & $\begin{array}{l}\text { Pos change } \\
\end{array}$ & Cam change & Cam change & Pos change & Pos change \\
\hline Cars & 20 & 22 & 23 & 20 & 20 & 20 & 19 & 12 \\
\hline P1X & $2.86 \pm 7.14$ & $2.14 \pm 6.61$ & $2.23 \pm 5.03$ & $0.57 \pm 6.56$ & $2.44 \pm 4.01$ & $1.87 \pm 5.56$ & $0.04 \pm 7.89$ & $0.16 \pm 6.30$ \\
\hline P1Y & $1.47 \pm 3.07$ & $0.84 \pm 3.27$ & $0.49 \pm 2.66$ & $1.09 \pm 2.52$ & $1.67 \pm 3.20$ & $0.68 \pm 3.21$ & $1.15 \pm 2.31$ & $0.31 \pm 3.21$ \\
\hline P2X & $1.62 \pm 4.57$ & $3.15 \pm 6.85$ & $0.93 \pm 4.17$ & $0.07 \pm 4.50$ & $1.37 \pm 4.13$ & $1.28 \pm 4.87$ & $0.37 \pm 4.87$ & $0.32 \pm 3.96$ \\
\hline P2Y & $3.08 \pm 3.04$ & $1.26 \pm 3.43$ & $0.47 \pm 2.85$ & $1.34 \pm 2.40$ & $0.63 \pm 3.27$ & & $1.29 \pm 3.19$ & $0.51 \pm 1.78$ \\
\hline P3X & $2.82 \pm 6.79$ & $0.62 \pm 6.42$ & $1.18 \pm 4.37$ & $0.12 \pm 6.02$ & $0.33 \pm 4.39$ & $2.38 \pm 4.72$ & $0.65 \pm 7.21$ & $0.61 \pm 5.74$ \\
\hline P3 Y & $3.95 \pm 2.91$ & $1.97 \pm 3.78$ & $0.90 \pm 2.94$ & $1.17 \pm 2.45$ & $0.37 \pm 2.98$ & $0.48 \pm 2.88$ & $1.17 \pm 3.54$ & $0.98 \pm 2.57$ \\
\hline P4Y & $0.07 \pm 3.13$ & $0.21 \pm 3.79$ & $0.26 \pm 2.98$ & $0.76 \pm 3.14$ & $1.61 \pm 4.26$ & $0.73 \pm 3.31$ & $0.96 \pm 3.42$ & $0.14 \pm 3.44$ \\
\hline P4Z & $2.74 \pm 2.78$ & $0.15 \pm 2.74$ & $0.33 \pm 2.64$ & $0.42 \pm 2.66$ & $0.55 \pm 3.53$ & $1.08 \pm 3.30$ & $0.04 \pm 2.30$ & $0.53 \pm 2.28$ \\
\hline
\end{tabular}

Table 5: Results for camera exchange and displacement tests.

the results show that the MBT and this concept to handle maintenance cases of the camera is suitable for the kind of automatic gap measurement application described in this paper. Camera exchanges or smaller position and rotation changes of the camera can be handled by the concept of measuring a test specimen with the MBT to correct calibrated relations of the system components. With this concepts a camera exchange can be performed within minutes, instead of some hours that are needed for initial measurements.

\section{CONCLUSION}

In this paper we presented the evaluation of modelbased tracking (MBT) for robotic gap measurements on painted cars. Different lighting situations in correlation with the car's gloss paint were evaluated and analyzed. In this context image and lighting optimizations were tested to maximize the matching quality of the MBT. In this regard, another evaluation considering the optimal CAD model selection for the MBT was presented. We showed the industrial use case of using MBT to track painted cars on a conveyor belt and to use the results to correct learned robot measurement points. With this method collisions between the robot and the cars can be avoided. The applicability of MBT for such an industrial use case was proven by an evaluation of the total accuracy of the system. Therefore, the gap measurement tool of the robot that consists of a laser line was used. It was shown that the majority of measurement points could be approached by the robot with an accuracy of up to $8 \mathrm{~mm}$. Furthermore, a method to exchange a calibrated camera in the system within minutes was presented. The method relies on a separate test specimen and was evaluated in detail. The results showed, that with this method an efficient camera maintenance model can be implemented, that merely introduces a small and acceptable error in regard of the total system accuracy. The presented methods and evaluations can help researchers and the industry to better assess and understand the influence and correlations of different factors on MBT in complex industrial use cases.

\section{REFERENCES}

[VP05] Lepetit, V., Fua, P. (2005). Monocular ModelBased 3D Tracking of Rigid Objects: A Survey. Foundations and Trends in Computer Graphics and Vision.
[Wue07] Wuest H., Wientapper F., Stricker D. (2007) Adaptable Model-Based Tracking Using Analysisby-Synthesis Techniques. In: Kropatsch W.G., Kampel M., Hanbury A. (eds) Computer Analysis of Images and Patterns. CAIP 2007.

[Com06] Comport, A., Marchand, E., Pressigout, M., Chaumette, F.: Real-time markerless tracking for augmented reality: the virtual visual servoing framework. IEEE Trans. on Visualization and Computer Graphics 12(4), 615-628 (2006)

[Sch20] Scheer,F., Neumann,M., Wirth,K., Ginader,M., Oezkurt,Y., Mueller,S.: Evaluation of model-based tracking and its application in a robotic production line. Journal of WSCG, 2020.

[Vac04] Vacchetti, L., Lepetit, V., Fua, P.: Combining edge and texture information for realtime accurate $3 \mathrm{~d}$ camera tracking. In: Proceedings of International Symposium on Mixed and Augmented Reality (ISMAR) (2004)

[Hin11] Hinterstoisser, S., Holzer, S., Cagniart, C., Ilic, S., Konolige, K., Navab, N.,Lepetit, V.: Multimodal Templates for Real-Time Detection of Texture-less Objects in Heavily Cluttered Scenes. IEEE International Conference on Computer Vision (ICCV), 2011

[Gar18] Garon,M., Laurendeau, D., Lalonde, J-F.: A Framework for Evaluating 6-DOF Object Trackers. In: Ferrari V., Hebert M., Sminchisescu C., Weiss Y. Computer Vision - ECCV 2018.

[Wu17] Wu, P-C., Lee, Y-Y., Tseng, H-Y., Ho, H-I., Yang, M-H., Chien, S-Y: [Poster] A Benchmark Dataset for 6DoF Object Pose Tracking. 186-191. 10.1109/ISMAR-Adjunct, 2017.

[Hod17] Hodaň,T., Haluza, P., Obdržálek, Š., Matas, J., Lourakis, M., Zabulis, X.: T-LESS An RGB-D Dataset for 6D Pose Estimation of Texture-less Objects, IEEE Winter Conference on Applications of Computer Vision (WACV), 2017

[Cre21] Creaform Metra Scan: https://www.creaform3d.com, 2021

[Far21] FARO Europe GmbH \& Co. KG, Faro arm, Faro laser tracker: https://www.faro.com, 2020

[Gom21] GOM GmbH, ATOS, https://www.gom.com, 2020. 